\title{
ACESSIBILIDADE AO SERVIÇO DE SAÚDE: PERCEPÇÕES DE USUÁRIOS E PROFISSIONAIS DE SAÚDE*
}

Leonardo Oliveira Leão e Silva ${ }^{1}$, Carlos Alberto Dias², Marina Mendes Soares ${ }^{3}$, Suely Maria Rodrigues ${ }^{4}$

\begin{abstract}
RESUMO: Estudo qualitativo, objetivou conhecer a percepção dos usuários e profissionais de saúde sobre os fatores que dificultam a acessibilidade à Estratégia Saúde da Família. As informações foram coletadas por meio de entrevista semiestruturada junto a 27 profissionais e 16 usuários de 4 Unidades com Estratégia Saúde da Família. Como método de análise das informações coletadas utilizou-se a Análise de Conteúdo de Bardin. Os resultados demonstraram que, na percepção dos profissionais e usuários, os principais empecilhos para o acesso ao serviço foram a localização da unidade, as características geográficas locais, o transporte, a delimitação inadequada do território de abrangência, a falta e o despreparo dos profissionais e, finalmente, a violência urbana. Nota-se que, embora a Estratégia Saúde da Família deva ser organizada com base na perspectiva territorial, na prática, o mapeamento da situação de saúde é realizado sem que se tenha uma compreensão do território em suas múltiplas peculiaridades.

PALAVRAS-CHAVE: Acesso aos serviços de saúde; Programa saúde da família; Territorialidade; Saúde Pública.

\section{THE ACCESSIBILITY OF THE HEALTH SERVICE: USERS' AND HEALTH CARE PROFESSIONALS' PERCEPTIONS}

ABSTRACT: This qualitative study aimed to understand how factors which hinder access to the Family Health Strategy are seen by health service users and health care professionals. The data was collected through semi-structured interviews with 27 health care professionals and 16 health service users of four Family Health Strategy units, and was analysed using Bardin's thematic content analysis. The results showed that in the perception of health service users and professionals alike, the principal obstacles to accessing the service were the unit's location, the local geographical characteristics, transport, inadequate clarification of the area covered by the unit, the lack or unpreparedness of the health care professionals, and - finally - urban violence. It is noted that although the Family Health Strategy is meant to be organized based on a territorial perspective, in practice the mapping out of the health situation is done without a full comprehension of areas' multiple characteristics.

KEYWORDS: Access to health services; Family health program; Territoriality; Public health.

\section{ACCESIBILIDAD AL SERVICIO DE SALUD: PERCEPCIONES DE USUARIOS Y PROFESIONALES DE SALUD}

RESUMEN: Estudio cualitativo, cuyo objetivo fue conocer la percepción de los usuarios y profesionales de salud acerca de los factores que dificultan la accesibilidad a la Estrategia Salud de la Familia. Las informacciones fueron recogidas por medio de entrevista semiestructurada con 27 profesionales y 16 usuarios de cuatro unidades con Estrategia Salud de la Familia. Como método de análisis de informaciones se utilizó la Análisis de Contenido de Bardin. Los resultados demostraron que, en la percepción de los profesionales y usuarios, los principales impedimientos para el acceso al servicio fueron la localización de la unidad, las características geográficas locales, el transporte, la delimitación inadecuada del territorio de abrangencia, la falta de preparación de los profesionales y, finalmente, la violencia urbana. Se concluye que, a pesar de la Estrategia Salud de la Familia ser organizada con base en la perspectiva territorial, en la práctica, el "mapeamento" de la situación de salud es realizado sin una comprensión del territorio en sus múltiplas singularidades. PALABRAS-CLAVE: Acceso a los servicios de salud; Programa Salud de la Familia; Territorialidad; Salud pública.

\footnotetext{
* Recorte da pesquisa "Portadores de Hipertensão Arterial Sistêmica: representações dos pacientes a respeito da doença", fomentada pela Fundação de Amparo à Pesquisa de Minas Gerais - FAPEMIG.

${ }^{1}$ Enfermeiro da Prefeitura Municipal de Governador Valadares - MG. Mestre em Gestão Integrada do Território.

${ }^{2}$ Psicólogo. Doutor em Psicologia Clínica. Professor do Programa de Pós-Graduação em Psicologia da Universidade Vale do Rio Doce. ${ }^{3}$ Acadêmica de Psicologia da Universidade Vale do Rio Doce. Bolsista de Iniciação Científica pela FAPEMIG.

${ }^{4}$ Cirurgiã Dentista. Doutora em Saúde Coletiva. Professora da Universidade Vale do Rio Doce.
}

Autor correspondente:

Leonardo Oliveira Leão e Silva

Prefeitura Municipal de Governador Valadares

Rua I, 398 - 35050-340 - Governador Valadares-MG-Brasil

E-mail: leooliveiraleao@hotmail.com
Recebido: $10 / 08 / 2011$

Aprovado: 28/10/2011

Cogitare Enferm. 2011 Out/Dez; 16(4):654-60 


\section{INTRODUÇÃO}

O Sistema Único de Saúde (SUS) exige, na organização dos serviços de saúde no Brasil, a existência de uma rede de atenção à saúde articulada que possibilite o acesso universal, integral, equânime, e o mais perto possível dos usuários. Esse acesso deve ser assegurado em todos os níveis de atenção, de acordo com a complexidade que cada caso requeira, e para, desse modo, satisfazer as necessidades dos usuários no tocante à saúde ${ }^{(1)}$.

O acesso da população à rede deve ocorrer através de uma porta de entrada, ou seja, para que aconteçam as ações de saúde é necessário o estabelecimento de um primeiro contato entre usuário e profissionais de saúde. Esse encontro deve ocorrer, principalmente, na atenção básica à saúde, tornando-se esta uma das principais portas para a assistência à saúde. Tal arranjo é apontado como caminho para a consolidação do SUS e tem sido apoiado e influenciado pela Organização Mundial da Saúde. De certa forma, esta realidade é determinante para a organização do SUS, mas para que ocorra, há que se garantir acessibilidade e acesso aos serviços de saúde ${ }^{(2)}$.

A acessibilidade está diretamente relacionada: às condições de vida, nutrição, habitação, poder aquisitivo e educação dos usuários; à presença de profissionais devidamente remunerados e preparados para o atendimento; à existência de instalações devidamente equipadas e inseridas em espaços nos quais usuários e profissionais possam interagir. A acessibilidade extrapola, portanto, a dimensão geográfica, abrangendo o aspecto econômico, a existência de um corpo técnico devidamente qualificado e a necessidade do usuário em buscar o serviço de saúde $^{(3)}$. Para que ela se efetive, deve ser discutida por todos os atores envolvidos nos serviços de saúde, já que todos devem ter condições necessárias para agir em prol da efetivação dessa acessibilidade.

Ainda, em relação a acessibilidade, deve-se considerar que os profissionais de saúde constituem-se em um fator que influencia de maneira considerável na busca dos usuários pelos serviços de saúde ${ }^{(4)}$. Diante disso, tais profissionais devem ter o perfil e estar qualificados para atender e resolver os principais problemas que demandam os usuários. Tal assertiva deixa clara a necessidade dos profissionais de saúde em conhecer os motivos que facilitam ou dificultam o acesso dos usuários ao serviço de saúde. Este conhecimento possibilitaria a intervenção rápida e ágil dos profissionais em direcionar os cuidados em saúde para um determinado grupo ou população em risco.

O conhecimento acima referenciado poderia ser obtido a partir da busca de informação, educação ou capacitação profissional. No entanto, mesmo havendo tal conhecimento, há que se buscar respostas à seguinte questão: quais são as percepções dos profissionais de saúde e dos usuários em relação às necessidades daqueles cadastrados pela Estratégia Saúde da Família (ESF), e que estratégias são empregadas pelos profissionais a fim de garantir o acesso aos serviços?

\section{METODOLOGIA}

Este estudo possui uma abordagem qualitativa, onde se buscou investigar diretamente com as pessoas envolvidas questões relativas à sua vivência para conhecer e compreender significados e práticas individuais e coletivas. Apresenta um referencial teórico, no campo da avaliação, que considera as dimensões de estrutura, de processo e de resultado, sendo focalizado nesse estudo a estrutura-processo do acesso de usuários à $\mathrm{ESF}^{(5)}$. Para tanto, foram realizadas 27 entrevistas semiestruturadas com profissionais de saúde pertencentes às equipes de Unidades de Saúde da ESF participantes, sendo 4 enfermeiras, 4 médicos, 3 técnicas em enfermagem e 16 agentes comunitárias de saúde. Além destes profissionais, participaram também 16 usuários cadastrados nestas unidades.

As entrevistas ocorreram individualmente nas dependências das Unidades da ESF, em ambiente reservado, favorecendo uma melhor comunicação entre entrevistado e entrevistando. A definição do número de indivíduos não se fez com base em amostragem probabilística, sendo este número delimitado pela saturação dos discursos ${ }^{(6)}$. As entrevistas foram realizadas no período de 27 de janeiro a 04 de abril de 2011, em quatro Unidades da Estratégia que compõem a rede de serviços da Secretaria de Saúde do município em que foi realizada a pesquisa.

Com o objetivo de observar fidelidade quanto ao registro das respostas fornecidas pelos profissionais de saúde e usuários, as entrevistas foram registradas com o auxílio de um gravador digital, com o consentimento prévio dos participantes. Tal registro viabilizou o retorno ao material, sempre que se fez necessário. Em todas as entrevistas manteve-se um caráter informal, a fim de que os profissionais de saúde e usuários se sentissem à vontade para relatar suas opiniões. Essas entrevistas foram transcritas pelos pesquisadores imediatamente após sua realização. 
A apuração dos dados foi realizada segundo a técnica da análise de conteúdo ${ }^{(7)}$. Tal análise proporcionou agrupar os dados em duas categorias de análise, a saber: Acessibilidade ao Serviço de Saúde e Atendimento ao paciente. No presente artigo será discutida mais profundamente a primeira categoria de análise e suas subcategorias. Os textos não sofreram correções linguísticas, preservando-se o caráter espontâneo das falas.

Para identificação e avaliação dos territórios de abrangência das ESF foram realizadas análises nos documentos fornecidos pela Secretaria Municipal de Saúde (SMS) referentes às demarcações e identificação dos locais de inserção das Unidades. Para identificação do território vivenciado pelos usuários, foram realizadas, durante um período de 10 dias para cada Unidade e respectivas áreas de abrangência, observações pelos pesquisadores.

O desenvolvimento do estudo respeitou todos os preceitos éticos que envolvem pesquisa com seres humanos estabelecidos pela Resolução 196/96 do Ministério da Saúde. Assim, foi solicitada autorização para a realização do estudo junto à SMS do município pesquisado e o projeto foi aprovado pelo Comitê de Ética em Pesquisa com Seres Humanos da Universidade Vale do Rio Doce (Parecer n. 002/2010). Todos os participantes do estudo assinaram o Termo de Consentimento Livre e Esclarecido. Com o intuito de preservar a identidade dos entrevistados, os fragmentos das entrevistas apresentados nesse trabalho foram identificados pelos termos "Profissional" e "Usuário", recebendo cada um o número correspondente ao da entrevista.

\section{RESULTADOS}

A partir da análise dos resultados foi possível dividir a categoria Acessibilidade ao Serviço de Saúde em cinco subcategorias: Território de abrangência; Localização das Unidades de ESF; Estrutura física; Profissionais de saúde; Violência urbana.

\section{Território de abrangência}

Ao criar uma Unidade de ESF o município deve respeitar as diretrizes impostas pelo Ministério da Saúde, dentre elas, a inserção da equipe em uma área cuja população tenha uma maior exposição a riscos sociais. Esta área não pode apresentar empecilhos de nenhuma ordem para o acesso à Unidade de Saúde pe- los usuários. Depois de adotadas estas recomendações e identificado o local para inserção da Unidade da ESF, ocorre, por parte da mesma ou da SMS, a definição de um território de abrangência, ou seja, uma área sob sua responsabilidade. Diante disso, os profissionais de saúde do presente estudo acreditam que os usuários devem respeitar o território da Unidade de Saúde e não os territórios que eles próprios vivenciam e praticam. Tais profissionais reconhecem isso como um problema e não como oportunidade de prestar o cuidado à saúde.

Eles [pacientes] não têm essa consciência de área de abrangência, de delimitação de área. Então, se eu tô passando mal aqui, eu quero ser atendido aqui [...]. Acaba tendo esses problemas [...]. Eles acham que têm que ser atendidos aqui. (Profissional 12)

Às vezes, a gente chega aqui, procura alguma pessoa aqui do Posto de Saúde, ai elas fala que não pode ser atendida, que o médico já foi embora. Então, é muita dificuldade mesmo. Tanto pros idosos quanto prás crianças e prá gente. Eu mesma quase nem consulto aqui porque eu não gosto de vir cá. Porque toda vez que eu venho eu passo raiva. Eu já procurei o posto. Lá eles falam com a gente, que tem que procurar o Posto de perto da residencia onde a gente mora. Ai não tem como atender. Diz eles que só atende da rua [...] pra lá. (Usuário 3)

Vale salientar que foram entrevistadas quatro equipes da ESF que atuam em duas estruturas físicas distintas. Por sua vez, cada uma dessas estruturas é compartilhada por duas equipes, cujas áreas de abrangência não são equidistantes. Este tipo de aproveitamento da estrutura física por duas equipes foi apontado como um problema para o acesso ao serviço, já que aumenta a distância de, pelo menos, uma equipe ESF do seu território de abrangência.

A maior dificuldade seria no sentido de localização do Posto. O Posto fica localizado fora da área de abrangência, então, a pessoa tem que caminhar pra chegar ao Posto. Às vezes demora quase meia hora prá chegar aqui. (Profissional 3)

Outro problema relatado pelos profissionais de saúde é o fato de existir usuários do serviço de saúde que não podem ser atendidos pela Unidade da ESF mais próxima de sua casa devido à divisão equivocada dos territórios de abrangência. 
Nós atendemos as pessoas que moram encostados no outro Posto. A maioria das pessoas mesmo, tão querendo sair daqui pra ir pra lá. Lá é mais próximo. (Profissional 4)

As "pessoas" citadas no discurso da entrevistada, ao procurarem a Unidade mais próxima de suas residências, tendem a receber diversas justificativas do serviço pelo não-atendimento como a que se segue:

Eles questionam muito isso e até, às vezes querem ser atendido aqui, mas a gente é obrigado a orientá-los que tem marcação de território e que temos que estar obedecendo para que não sobrecarreguem nem uma e nem outra estratégia. Então, procuramos orientar eles nesse sentido. (Profissional 14)

A conduta do profissional de saúde, possivelmente condicionada pela supervisão, baseada no território de abrangência e relacionada à organização da Unidade da ESF, cria obstáculos para o paciente. Este fica irritado, frustrado e decepcionado por não receber o atendimento desejado no momento de sua necessidade:

Ah, eu vou embora xingando, com raiva. Por quê? Igual meu menino de sete anos tava com febre, precisando consultar aqui. Quando eu chego aqui, ai eles viraram e falaram assim que a enfermeira já tinha ido embora e o médico também. Eles não poderiam fazer nada. Era para levar para o hospital. Quando a gente chega ao hospital eles viram para a gente e falam que não é urgência. É pequeno serviço. Então, tinha que ser atendido no Posto perto da residência da gente. (Usuário 6)

\section{Localização das ESF}

Os usuários e profissionais de saúde identificam a localização geográfica dos serviços como um dos fatores que interfere na acessibilidade:

Alguns pacientes têm dificuldade de acesso. Então a questão do morro, paciente idoso tem dificuldade de tá vindo à Unidade [...]. (Profissional 1)

Outro ponto relatado pelos profissionais de saúde foi a forma de deslocamento utilizada pelos usuários. Segundo eles, esta é uma variável que facilita ou dificulta o acesso ao serviço de saúde, considerando que a forma de deslocamento frequentemente utilizada pelos usuários para o alcance da Unidade da ESF é a pé. Por um lado, ela facilita por ser a mais prática e também a mais econômica forma de locomoção. No entanto, ela não pode ser utilizada adequadamente pelos usuários devido à distância e à característica geográfica do local de inserção das Unidades de Saúde. Trata-se de uma região cujas vias são caracterizadas por fortes aclives e declives que colocam em risco o bem-estar de alguns pacientes, sobretudo de idosos. Outro ponto que atua como empecilho no deslocamento ao serviço de saúde é a oferta de linha de ônibus público gratuito para idosos nos bairros onde estão inseridos os serviços da ESF. Para eles, fica mais fácil ir ao Pronto Socorro Municipal, já que o ônibus tem um ponto de parada em frente ao mesmo, enquanto para alcançar a Unidade da ESF, os pacientes devem caminhar aproximadamente quatro quarteirões em aclive depois de deixar o ônibus:

Tem morro, e muito! Tem que vir de ônibus. A minha mulher, eu não tô podendo subir morro nem descer com ela. Eu tô operado recente. Recente assim, eu operei em janeiro, mas minha operação foi longa, então estou prosseguindo o tratamento lá no hospital. (Usuário 5)

\section{Estrutura física}

Além da distância entre as residências dos usuários e a Unidade da ESF, a própria estrutura física desta foi apontada como um complicador para o paciente, já que são construções velhas, adaptadas, pouco iluminadas, organização de espaço e de ambientes inadequados, umidade e falta de privacidade:

[...] a estrutura física, que não tem condição nenhuma estrutural. Esse ESF é um local assim adaptado, que é uma coisa doada pela igreja e foi feito as adaptações. (Profissional 5)

\section{Profissionais de saúde}

Observou-se a existência de retornos constantes de usuários às Unidades avaliadas devido à falta constante de profissionais médicos. Tal panorama é citado como uma dificuldade para o paciente dar continuidade ao atendimento, dificultando, desta forma, o acesso:

Então, às vezes, dá confusão. O paciente pergunta: 'Ah! Mas porque só tem um médico aqui?' Hoje mesmo aconteceu isso aqui. Paciente chegou e falou: 'quero ser atendida'. Foi até a estratégia dois. 'Ah eu quero 
ser atendida'. O médico hoje não está, o médico tirou uns dias de férias. 'E porque aqui não tem médico?' Falei: 'aqui minha senhora, aqui não é uma Unidade de plantão de saúde. Aqui é uma Estratégia Saúde da Família'. (Profissional 4)

A paciente foi em busca de uma atenção que não aconteceu e ela ainda não recebeu uma justificativa adequada para resolução de sua angústia. Estes pacientes, devido à necessidade de cuidados são forçados a procurar outro local para receber atendimento, e a primeira escolha é o Pronto Socorro Municipal.

Venho andando. Moro pertinho do posto. Já aconteceu de eu vir aqui, não ser atendida, eu fui lá para o [Hospital] Regional. Não atendia a gente na mesma hora e a minha menina precisava. Aí, eu ia lá para o centro. (Usuário 9)

\section{Violência urbana}

A violência urbana foi percebida como um dificultador do acesso às Unidades da ESF avaliadas, já que foram citados casos de pacientes que não têm acesso ao serviço devido a "atritos" com gangues ou dívidas de drogas:

Na hora que eu entrei na casa dela, ela falou: 'Doutor, vai embora porque daqui a cinco minutos vai começar o tiroteio aqui. O senhor tá vendo a polícia descendo do lado ali?' Aí, eu corri! [...]. (Profissional 22)

De certa forma, o território da ESF e o território da violência são diferenciados. A administração pública, por considerar este último como marginal, não o reconhece como importante ao criar projetos de implantação das Unidades da ESF. O efeito disso é a inserção de Unidades em locais de conflitos territoriais, portanto, locais impróprios ao alcance dos objetivos da Estratégia.

\section{DISCUSSÃO}

A ESF possui foco no atendimento à saúde das famílias, incluindo-se atividades de promoção de saúde, e também sobre os ambientes de reprodução social delas. O menor território do sistema de saúde (a família) possui como vantagem a possibilidade de captar dados demográficos, epidemiológicos, e de condições de vida, incluindo as ambientais. Daí a importância estratégica do conhecimento do território para os profissionais de saúde, já que sua análise favorece a consolidação de ações de saúde para o enfrentamento de problemas e necessidade da população ${ }^{(8-9)}$. No entanto, o mero conhecimento do território adscrito ao serviço ou a disponibilidade deste serviço não implica que esteja garantido o acesso do usuário, embora o favoreça.

O reconhecimento desse território é um passo inicial para caracterizar a população e seus problemas de saúde, bem como para avaliar o impacto dos serviços sobre os níveis de saúde dessa população. Ou seja, o território é a consequência de uma acumulação de situações históricas, ambientais e sociais que promovem condições particulares para a produção de doenças ${ }^{(10)}$. No entanto, durante a análise dos discursos dos profissionais de saúde, observou-se que a falta de conhecimento do território, bem como das territorialidades onde as Unidades estão inseridas, provocam problemas que dificultam o acesso da população ao serviço ${ }^{(11)}$.

Dentre os problemas apontados pelos profissionais e usuários está a localização da Unidade de Saúde. Tal situação também é observada por outros autores ${ }^{(3,15)}$, que identificam a necessidade de revisão do atual desenho da territorialização das Unidades de Saúde com vistas a uma melhor organização da assistência. Esse novo olhar deve ser pautado na percepção do território como processo dinâmico de relações e de expressão do processo saúde-doença ${ }^{(10)}$.

O território, compreendido em suas múltiplas vertentes, sofre transformações provenientes de relações de poder ${ }^{(12)}$, formando-se dentro de vários territórios. Tal fenômeno se dá, principalmente, nas grandes cidades, a exemplo do território da prostituição; do narcotráfico; do homossexualismo; das gangues e outros que podem ser temporários ou permanentes ${ }^{(13)}$. Diante disso, observa-se a necessidade de se conhecer as múltiplas territorialidades existentes em um espaço geográfico antes da inserção das Unidades da ESF. Já que os territórios de certos grupos podem prejudicar, através do aumento da violência urbana, a acessibilidade ao serviço de saúde. Desta forma, dificulta-se o cuidado e a continuidade dos tratamentos, gerando graves e complexas consequências à qualidade de vida, à atenção da saúde e à prevenção das doenças na população ${ }^{(14)}$.

A Atenção Básica por ser a principal porta de entrada, tem um papel fundamental na resolutividade das demandas dos usuários, bem como, na realização de procedimentos necessários, a fim de promover a reabilitação da saúde ${ }^{(16)}$. Quando esta não se efetiva 
ocorrem problemas em relação ao acesso e ao atendimento, impondo aos usuários o retorno frequente aos serviços de saúde ${ }^{(17)}$. Tal panorama foi observado nas Unidades da ESF avaliadas, e como consequência, os usuários vão à busca do hospital para atender a sua necessidade de saúde. Tal perspectiva contribui para a manutenção do imaginário popular, segundo o qual o Pronto Socorro é o local que resolve todos os problemas de saúde ${ }^{(18)}$.

Todo o panorama demonstrado deve ser considerado na análise do acesso aos serviços, já que esta é considerada uma etapa inicial a ser vencida pelo usuário quando parte em busca da satisfação de uma necessidade de saúde. No entanto, percebeu-se que o sucesso desta etapa inicial é dificultado pela própria organização do serviço de saúde.

Sugere-se que seja realizada uma análise primária de todo o território bem como das territorialidades, antes da adoção da ESF numa determinada região. Tal procedimento pode garantir uma melhor localização geográfica, facilidade de transporte, diminuição de queixas em relação ao território de abrangência e, sobretudo, redução das crescentes interferências promovidas pelo território do crime, em especial nas médias e grandes cidades. Tal análise constitui-se, a priori, um facilitador do acesso da população ao serviço de saúde, quando o projeto de implantação da ESF busca a melhoria da acessibilidade, voltada para a organização da oferta e pautando-se na perspectiva de mudança do modelo assistencial ${ }^{(19)}$.

\section{CONSIDERAÇÕES FINAIS}

Observou-se que, tanto na percepção dos usuários quanto na dos profissionais de saúde, as principais barreiras ao acesso ao serviço são: o transporte inadequado, a falta e despreparo dos profissionais, a violência urbana, a localização imprória das Unidades da ESF, as características geográficas locais e a delimitação inadequada do território de abrangência. Nota-se que, embora a ESF seja organizada com base na perspectiva territorial, na prática, o mapeamento da saúde tem sido realizado sem a compreensão do território em suas múltiplas peculiaridades.

O conhecimento dos profissionais acerca dos fatores que dificultam o acesso da população ao serviço de saúde mostra-se importante. No entanto, a posse desse conhecimento, por si só, não garante uma efetiva atenção às necessidades de cuidado da população. Para que isto ocorra, faz-se necessário que o profissional assuma uma postura mais ativa, tanto no atendimento ao usuário quanto no planejamento de saúde de seu município.

\section{REFERÊNCIAS}

1. Ministério da Saúde (BR). Secretaria Nacional de Assistência à Saúde. ABC do SUS: doutrinas e princípios. Brasília: Ministério da Saúde; 1990.

2. World Health Organization (WHO). The world health report 2008 - primary health care (now more than ever). Geneva: World Health Organization; 2008.

3. Silva JES, Medina MG, Aquino R, Fonseca ACF, Vilas-bôas ALQ. Acessibilidade geográfica à atenção primária à saúde em distrito sanitário do município de Salvador, Bahia. Rev Bras Saude Mater Infant. 2011;10 Suppl 1:49-60.

4. Fekete MC. Estudo de acessibilidade na avaliação dos serviços de saúde. In: Santana JP, organizador. Desenvolvimento gerencial de unidades básicas do Sistema Único de Saúde (SUS). Brasília: OPS; 1997. p.5-7.

5. Donabedian A. La calidad de la atención médica: definición y métodos de evaluación. Cidade do México: Ediciones Copilc; 1984.

6. Bauer MW, Aarts B. A construção do corpus: um princípio para a coleta de dados qualitativos. In: Bauer M, Gaskell G, organizadores. Pesquisa qualitativa com texto, imagem e som. Petrópolis: Vozes; 2002. p. 39-63.

7. Bardin L. Análise de conteúdo. $20^{\mathrm{a}}$ ed. Lisboa: Edições 70; 2009.

8. Barcellos C, Sabroza PC, Peiter P, Rojas LI. Organização espacial, saúde e qualidade de vida: a análise espacial e o uso de indicadores na avaliação de situações de saúde. Informe Epidemiológico do SUS. 2002;11(3):129-38.

9. Mendes EV. A construção social da vigilância à saúde do distrito sanitário. Brasília: OPS. Série Desenvolvimento de Serviços de Saúde; 1993.

10. Monken $\mathrm{M}$, Barcellos $\mathrm{C}$. Vigilância à saúde e território utilizado: possibilidades teóricas e metodológicas. Cad Saúde Pública. 2005;21(3):898-06.

11. Sack RD. Human territoriality. Cambridge: Cambridge University Press; 1986.

12. Raffestin C. Porumageografia dopoder. São Paulo: Ática; 1993. 
13. Souza MJL. O território: sobre espaço e poder: autonomia e desenvolvimento. In: Castro IE, Gomes PCC, Corrêa RL, organizadores. Geografia: conceitos e temas. Rio de Janeiro: Bertrand Brasil; 2001.

14. Franco S, Suarez CM, Naranjo CB, Báez LC, Rozo P. Efeitos do conflito armado sobre a vida e a saúde na Colômbia. Ciênc Saúde Coletiva. [Internet] 2010;11 Suppl.0 [acesso em 15 jul 2011]. Disponível: http:// dx.doi.org/10.1590/S1413-81232006000500014

15. Sousa MF. O Programa Saúde da Família no Brasil: análise do acesso à atenção básica. Rev Bras Enferm. 2008;61(2):153-8.

16. Oliveira MM, Coimbra VCC, Kantorski LP, Jardim VMR, Heck RM, Ceolin T. Acesso dos usuários de uma unidade de Saúde da Família a serviços complementares. Cogitare Enferm. 2009;14(1):30-6.

17. Gomes FM, Silva MGC. Programa Saúde da Família como estratégia de atenção primária: uma realidade em Juazeiro do Norte. Ciênc Saúde Coletiva. 2011;16 Suppl $1: 893-902$.

18. Cecilio LCO. Modelos tecno-assistenciais em saúde: da pirâmide ao círculo, uma possibilidade a ser explorada. Cad Saúde Pública. 1997;13(3):469-78.

19. Cunha ABO, Vieira-da-Silva LM. Health services accessibility in a city of northeast Brazil. Cad Saúde Pública. 2010;26(4):725-37. 